\title{
Expression of Recombinant Human Coagulation Factor VII by the Lizard Leishmania Expression System
}

\author{
Sina Mirzaahmadi, ${ }^{1}$ Golnaz Asaadi-Tehrani, ${ }^{1}$ Mojgan Bandehpour, ${ }^{2}$ \\ Nooshin Davoudi, ${ }^{3}$ Leila Tahmasbi, ${ }^{4}$ Nahid Hosseinzadeh, ${ }^{2}$ Hasan Mirzahoseini, ${ }^{3}$ \\ Kazem Parivar, ${ }^{1}$ and Bahram Kazemi ${ }^{2,5}$ \\ ${ }^{1}$ Department of Biology, Science and Research Branch, Islamic Azad University, Tehran, Iran \\ ${ }^{2}$ Cellular and Molecular Biology Research Center, Shahid Beheshti University of Medical Sciences, Tehran, Iran \\ ${ }^{3}$ Biotechnology Department, Pasteur Institute, Tehran, Iran \\ ${ }^{4}$ Department of Hematology, Faculty of Paramedical Sciences, Shahid Beheshti University of Medical Sciences, Tehran, Iran \\ ${ }^{5}$ Biotechnology Department, Faculty of Medicine, Shahid Beheshti University of Medical Sciences, Tehran, Iran \\ Correspondence should be addressed to Bahram Kazemi, bahram_14@yahoo.com
}

Received 19 March 2011; Revised 28 April 2011; Accepted 17 June 2011

Academic Editor: Michael Kalafatis

Copyright () 2011 Sina Mirzaahmadi et al. This is an open access article distributed under the Creative Commons Attribution License, which permits unrestricted use, distribution, and reproduction in any medium, provided the original work is properly cited.

\begin{abstract}
The variety of recombinant protein expression systems have been developed as a resource of FVII gene expression. In the current study, the authors used a novel protein expression system based on the Iranian Lizard Leishmania, a trypanosomatid protozoan as a host for expression of FVII. Plasmid containing cDNA encoding full-length human FVII was introduced into Lizard Leishmania and positive transfectants were analyzed by SDS-PAGE and Western blot analysis. Furthermore, biological activity of purified protein was detected by PT assay. The recombinant strain harboring a construct was analyzed for expression of FVII at the mRNA and protein level. Purified rFVII was obtained and in order to confirm the purified compound was in fact rFVII. Western blot analysis was carried out. Clotting time in PT assay was reduced about 30 seconds with the purified rFVII. In Conclusion, this study has demonstrated, for the first time, that Leishmania cells can be used as an expression system for producing recombinant FVII.
\end{abstract}

\section{Introduction}

FVII is a vitamin-K-dependent serine protease synthesized in the liver and plays an important role in blood coagulation [1]. The gene for human FVII (NG_009262.1) is located on chromosome 13 (13q34); it has approximately $14 \mathrm{~kb}$ and consists of nine exons and eight introns. Alternative splicing patterns of the gene mRNA transcripts result in 2 coagulation factor VII isoforms, "a" and "b" precursors. Isoform "b" (NM_019616.2) is the prevailing form in normal liver; it does not include exon $1 \mathrm{~b}$ and thus encodes a shorter signal peptide than isoform "a" (NM_000131.3). The mature protein encoded by the two transcript variants is identical with a single-chain peptide with 406 amino acid residues and molecular mass of approximately $50 \mathrm{KDa}$ [2].

Upon activation, FVII is converted to the protease FVIIa, and a peptide bond between $\operatorname{Arg} 152$ and Ile 153 is separated.
So, the light chain, consisting of a $\gamma$-carboxyglutamic-acid containing (Gla) domain and two epidermal growth-factorlike (EGF) domains, is covalently connected to the heavy chain (the serine protease domain) through a single disulfide bond [3] (Figure 1).

Based on the understanding of the role of activated FVII in the treatment of the coagulation disorders, synthesis of recombinant activated FVII (rFVIIa) was considered. A review of application of rFVIIa (Novo Seven) over the past two decades indicates that initially this compound was approved for use in patients with congenital or acquired hemophilia who produce inhibiting antibodies toward factor VIII or IX. By this approach, high concentrations of FVIIa, which are achieved by pharmacological doses of rFVIIa, bind to preactivated platelets. Through this approach, it can bypass the need for VIIIa and IXa as a result of activation of FX to FXa. This would result in an increased generation 


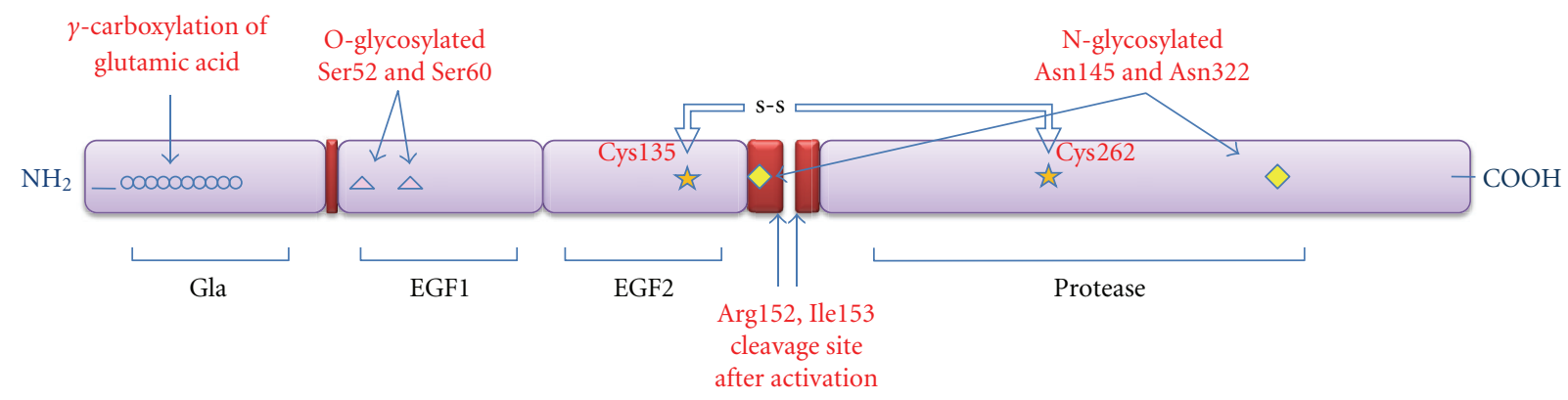

FIgURE 1: Human coagulation factor VII. FVII is a serine protease of 406 residues. Different domains of FVII are shown. Posttranslational modifications of the FVII molecule include (1) $\gamma$-carboxylation of 10 glutamic acid residues in the N-terminal part of the molecule, (2) N-glycosylation of asparagine residues in positions 145 and 322, and (3) O-glycosylation of serine residues 52 and 60.0 , gammacarboxyglutamic acid; $\triangle$, O-glycosylation sites; $\diamond, \mathrm{N}$-glycosylation sites; Arg152 and Ile153, cleavage site generated after activation; $\grave{\star}$, joining of two chains of FVIIa by a single disulfide bond between cys135 and cys262.

of thrombin which produces a tight, well-structured fibrin haemostatic plug, which is resistant to fibrinolysis [4-6].

Also, it is reported in the literature that the recombinant factor VIIa has been used effectively in patients with other categories of coagulation defects or patients with normal coagulation system who, for instance, experience excessive bleeding, as a result of trauma or surgery. However, it should be mentioned that adverse events such as arterial thromboembolism have been reported among patients who received rFVIIa, particularly among those who were 65 years of age or older [7-9].

Initially, BHK cells and subsequently the variety of recombinant protein expression systems such as insect and mammalian cells have been developed as a resource of FVII gene expression $[4,10,11]$.

Using mammalian systems has a number of drawbacks: (a) high price of cell-culture media, (b) possibility of contamination of products with viruses and prions depending on the components of animal origin, and (c) high purification cost $[12,13]$.

Recently, a novel protein expression system was introduced which was based on the nonpathogenic trypanosomatid flagellate Leishmania tarentolae, a protozoan parasite of lizards [14].

The main advantages of Leishmania expression system are as follows:

(1) easy to handle like E. coli and yeast expression systems,

(2) full eukaryotic protein folding,

(3) The mammalian-type posttranslational modification of target proteins and cultivation in low-cost media,

(4) high specific growth rate in comparison to mammalian cells $[15,16]$.

In the present study, attempt is made to express heterologous recombinant human FVII (rFVII) by the Iranian Lizard Leishmania (I.L.L.) Expression System [17].

\section{Materials and Methods}

2.1. Cell Line, Hosts, and Plasmid. Human hepatoma HepG2 cell line [18] was obtained from the National Cell Bank of Iran (ncbi code: c158). It was grown in $\mathrm{RPMI}_{1640}$ (Gibco, 52400: U.K.) medium at $37^{\circ} \mathrm{C}$ in $5 \% \mathrm{CO}_{2}$ supplemented with (a) $10 \%$ fetal bovine serum, (b) $1 \mu \mathrm{g} / \mathrm{mL}$ vitamin $\mathrm{K} 1$, (c) $100 \mathrm{u} / \mathrm{mL}$ penicillin and) (d) $100 \mu \mathrm{g} / \mathrm{mL}$ streptomycin (GIBCO, Pen-Strep,15140). The E. coli Top10 strain, the bacterial host, was cultured in LB broth medium. pLEXSYhyg2 plasmid was prepared from Jena Bioscience company of Germany.

2.2. Preparation of FVII cDNA. Total RNA was extracted using a commercial kit (QIAGEN RNeasy Mini Kit, Germany) from the hepG2 cell line. The synthesis of the FVII encoding cDNA was performed by using SuperScript III reverse transcriptase (Invitrogen, USA). RT reaction was carried out at $25^{\circ} \mathrm{C}$ for $10 \mathrm{~min}, 55^{\circ} \mathrm{C}$ for $50 \mathrm{mins}$ and $85^{\circ} \mathrm{C}$ for 5 min using random hexamer.

The PCR program consisted of an initial denaturation at $94^{\circ} \mathrm{C}$ for 5 minutes. A total of 35 cycles was carried out comprising of the following steps: $94^{\circ} \mathrm{C}$ for $30 \mathrm{~s}$, $60^{\circ} \mathrm{C}$ for $60 \mathrm{~s}, 72^{\circ} \mathrm{C}$ for $60 \mathrm{~s}$, followed by $72^{\circ} \mathrm{C}$ for $5 \mathrm{~min}$ as a final extension using the forward primer $5^{\prime}$ CTCGAGATGGTCTCCCAGGCCCTCAG3' and reverse primer 5'GCGGCCGCCTAGGGAAATGGGGCTCGCAG3'.

2.3. Plasmid Construction and Confirmation of the Prepared Construct. The purified PCR product of transcript isoform "a" was ligated to pTZ57R/T vector [19] and transformed in E. coli TOP10 strain competent cell as de-scribed previously [20]. The presence and accuracy of the insert was confirmed by restriction analysis and heminested PCR using two pairs of internal primers, forward primer 5'ATGGTCTCCCAGGCCCTCAG3' and reverse primer $5^{\prime}$ AGATGGTGTTGATCAGGGTCC 3 ' flanking a $700 \mathrm{bp}$ fragment inside the gene, and final verification was carried out using DNA sequencing.

The cloned FVII gene was digested by NotI and SlaI restriction enzymes (Fermentas, Lithuania) and cloned into pLEXSY-hyg2 plasmid (EGE-232, Jena Bioscience, Jena, 
Germany). The presence and correct orientation of the insert was analyzed by PCR and NotI and SlaI restriction enzymes.

2.4. Transfection and Cultivation Condition of Lizard Leishmania. I.L.L. [17] was cultivated in RPMI1640 (GibcoBRL, Eggenstein, Germany) medium supplemented with $10 \%$ fetal bovine serum, $100 \mathrm{u} / \mathrm{mL}$ penicillin and $100 \mu \mathrm{g} / \mathrm{mL}$ streptomycin (GIBCO, Pen-Strep, 15140), at $25^{\circ} \mathrm{C}, 15 \mu \mathrm{g} / \mathrm{mL}$ haemin (Sigma, St. Louis, Mo, USA). Plasmid containing cDNA encoding human FVII was linearized with SwaI (BIORON, Germany) [14] and used for transfection of the I.L.L. by electroporation in $4 \mathrm{~mm}$ cuvettes using an electroporator (Bio-Rad Gene Pulser) [21]. After two pulses, stable transfectants were selected on solidified RPMI media containing $25 \mu \mathrm{g} / \mathrm{mL}$ hygromycin B (Sigma, St. Louis, Mo, USA) [22]. For transcription and expression assays, recombinant I.L.L were cultured in RPMI-based suspension supplemented with haemin $(5 \mu \mathrm{g} / \mathrm{mL})$, penicillin $(50 \mathrm{U} / \mathrm{mL})$, and streptomycin $(50 \mu \mathrm{g} / \mathrm{mL})$ at $26^{\circ} \mathrm{C}$ in the absence of light and under shaking condition (140 rpm) $[22,23]$.

2.5. Expression and Purification of Recombinant FVII. The expression of rFVII was assayed by RT-PCR reaction on extracted total RNA of approximately $10^{8}$ recombinant Leishmania cells. Amplifications of $1400 \mathrm{bp}$ of the FVII fragment were carried out by specific primers. Furthermore, the expression of rFVII protein in Leishmania was confirmed by SDS-PAGE and western blotting procedure.

To select positive transfectants of I.L.L., cells were harvested by centrifugation at $3000 \mathrm{rpm}$ for $15 \mathrm{~min}$ and washed in PBS. The pellets were immediately lysed in $2 x$ SDSPAGE sample buffer (100 mMTris-HCl pH 8, 20\% glycerol, $4 \%$ SDS, $2 \%$ beta mercaptoethanol, $0.2 \%$ bromophenol blue) then boiled for 5 minutes. Samples were loaded on a $12 \%$ SDS-PAGE. The gel was stained by Coomassie brilliant blue R250, and gene expression was analyzed. The same procedures were carried out on the control sample in parallel for comparison.

For Western blot analysis, cell lysate was separated on $12 \%$ SDS-PAGE and electrophoretically transferred onto pvdf membrane. After UV crosslinking for protein fixation, the membrane was blocked with 3\% BSA at room temperature. The specific factor VII polyclonal antibody, HRP-conjugated (US Biological, F0015-20B) was used in 1:500 dilutions. The protein band was detected by DAB (Diaminobenzoic Acid) and $\mathrm{H}_{2} \mathrm{O}_{2}$.

After selecting the appropriate clone and cell growth, cell pellet from $10 \mathrm{~mL}$ shake flask culture was obtained with centrifuge in $3000 \mathrm{rpm}$ for $10 \mathrm{~min}$ and then resuspended in $5 \mathrm{mM}$ Tris- $\mathrm{HCl}, \mathrm{pH}$ 8.2. The suspension was immediately sonicated twice for $20 \mathrm{~s}$. Then, cell lysate was applied to an affinity column containing specific factor VII polyclonal antibody coupled to $\mathrm{CNBr}$-activated sepharose $4 \mathrm{~B}$ resin (Amersham Biosciences, Sweden). According to the manufacturer's instructions, rFVII was eluted with the changes in $\mathrm{pH}$ and by using $0.1 \mathrm{M}$ glycine, with the final $\mathrm{pH}$ of 2.8 . Then, acetone precipitation of adsorbed rFVII was performed. The precipitated product was resuspended in 1x TBS, and dot blot analysis was carried out by spotting the suspension

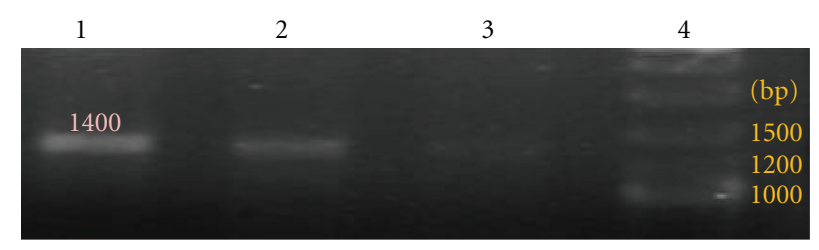

(a)

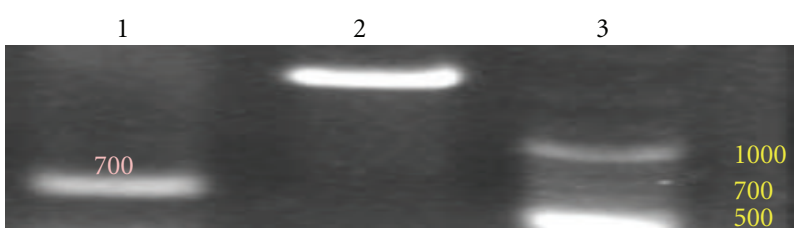

(b)

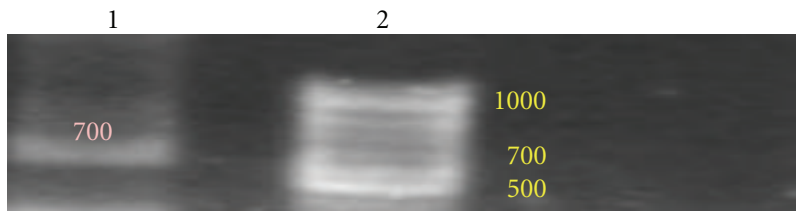

(c)

Figure 2: (a) FVII-PCR product, Lanes (1, 2, 3), 100 bp DNA ladder (4) (b) Restriction analysis of PCR product. Lane 1: digestion with HphI that revealed a $700 \mathrm{bp}$ fragment, Lane 2: PCR product without enzyme effect. (c) Lane 1: 700-bp PCR product obtained by heminested PCR.

onto a pvdf membrane. The specific factor VII polyclonal antibody was allowed to bind, and positive-colored spot was detected.

Subsequently, the glycine buffer composition of eluted rFVII was exchanged by $0.1 \mathrm{M}$ imidazole, $\mathrm{NaCl} 0.2 \mathrm{M}$, pH 7.3 buffer through the dialysis.

After Dialysis, soluble protein was further characterized by SDS-PAGE and western blot analysis, which was performed as mentioned above. Also, purified rFVII concentration was determined spectrophotometrically by Biophotometer (Eppendorf, Germany) at $280 \mathrm{~nm}$.

2.6. Analysis of Purified rFVII. For determination of coagulant activity of purified rFVII, clotting times of the dialyzed rFVII were obtained by a one stage clotting assay (PTbased assay for FVII). Hence, PT assay was performed based on human plasma immunodepleted of factor VII (Diagnostica Stago, France) in combination of purified rFVII and human thromboplastin-D (Thermo Scientific, USA). The coagulation was initiated by addition of PT reagent, and the coagulation time was recorded and compared with that of FVII (FVIIa, US Biological), as a positive control and purified sample obtained from untransfected I.L.L. (negative control), using fibrin timer cups and a semiautomated coagulation analyzer (Option 4 plus Biomerieux, Germany).

A standard curve was made, by using dilutions of $(1 / 1)$, $(1 / 2),(1 / 5),(1 / 10),(1 / 20),(1 / 40)$ of Unicalibrator (Stago, Ref. 00625) that related to defined $100 \%, 50 \%, 20 \%, 10 \%$, $5 \%$, and $2.5 \%$ of FVIIa levels, respectively. Also, several dilutions of dialyzed protein were made. An aliquot $(100 \mu \mathrm{L})$ 


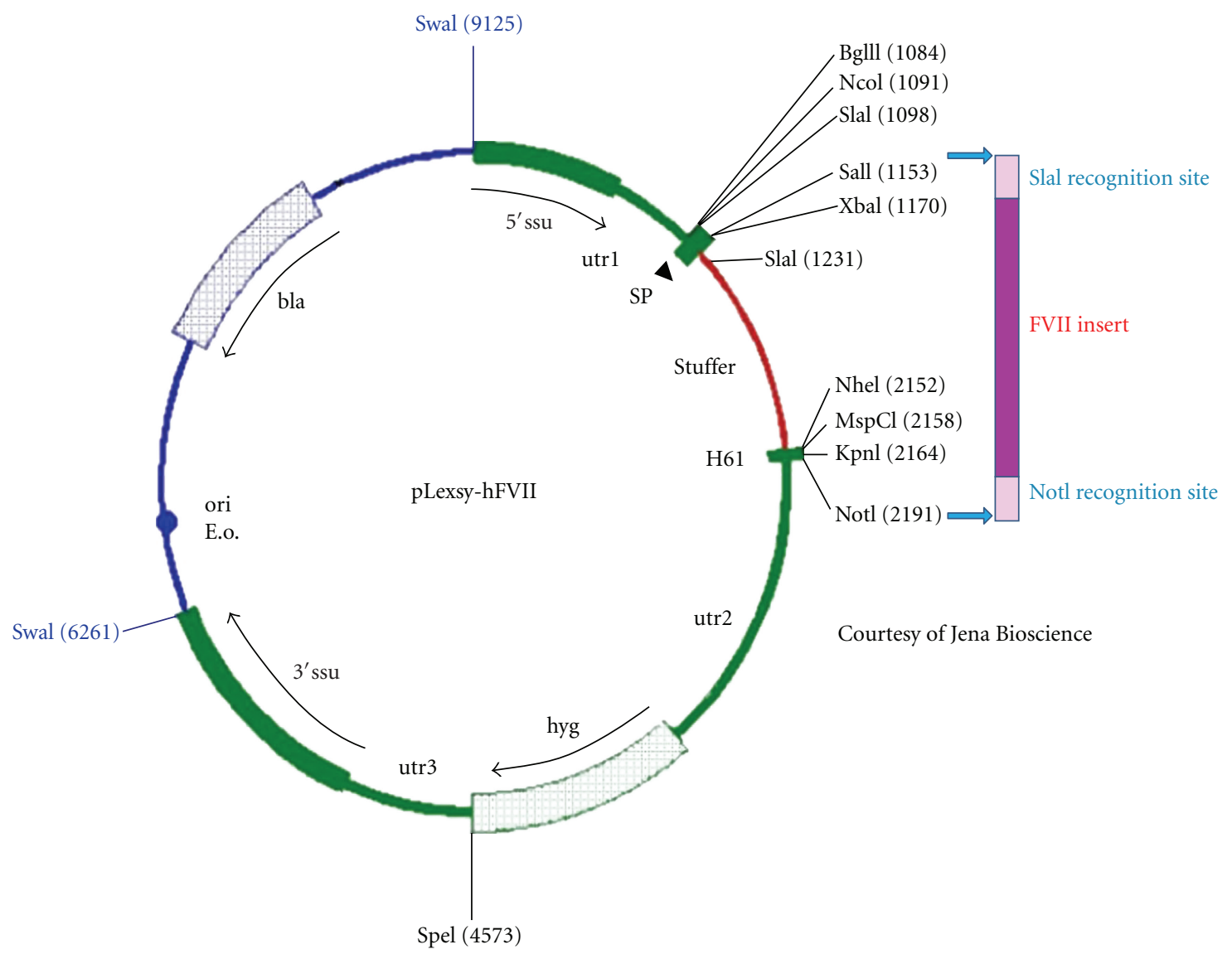

FIGURE 3: Construction of expression plasmid containing human FVII (pLexsy-hFVII). Map of pLEXSY-2 vector and the strategy used for plasmid construction are shown. The cDNA sequence encoding human FVII was inserted in corresponding restriction sites SlaI and NotI. More information about the vector itself can be found on http://www.jenabioscience.com/, pLEXSY-hyg2 (EGE-232).

of each diluted sample was added to $100 \mu \mathrm{L}$ of human plasma immunodepleted of factor VII; $200 \mu \mathrm{L}$ of thromboplastin was added and the clotting time was recorded immediately [24]. All determinations were carried out in duplicate and were compared to the standard curve. Hence, specific activity of the purified rFVII was determined.

\section{Results}

3.1. FVII Fragments Preparation and Its Confirmation. Specific primers were used for the isolation of full-length rFVII, and the amplicon with expected size (about 1400bp) was produced (Figure 2(a)). In order to confirm the correctness of the amplicon, purified PCR product was digested with $H p h I$ which resulted in two fragments of 700bp (Figure 2(b)).

3.2. Construction of the Recombinant pLEXSY-hyg2-FVII Plasmid. To generate pTZ57r-FVII, the PCR product was cloned into pTZ57r vector. The accuracy of the gene was determined by heminested PCR approach which yielded the specific 700-bp amplified product (Figure 2(c)), and by KpnI enzyme digestion which revealed a 1200-bp fragment. Additional confirmation was obtained by DNA sequencing.
Subsequently, the resultant pTZ57r-FVII was digested with SlaI and NotI restriction enzymes. In order to generate pLexsy-hFVII construct, the correct fragment was inserted into the corresponding restriction sites of pLEXSY-hyg2 expression vector (Figure 3 ).

3.3. Expression and Purification of Recombinant Human FVII in I.L.L. Cells. Approximately $5 \mu \mathrm{g}$ pLEXSY-hyg2-FVII plasmid was linearized by SwaI restriction enzyme (Figure 4(a)). It was transfected into I.L.L. cells by electroporation, and stable transfectants were selected by culturing cells on solid medium with hygromycin. Subsequently, two to three passages in liquid medium containing hygromycin were performed, and the recombinant promastigotes harboring a construct was analyzed for expression of rFVII.

In order to verify rFVII mRNA synthesis in the host, RT-PCR was performed on total RNA extracted from transformed cells using FVII primers. The desired band with the expected size of $1400 \mathrm{bp}$ was obtained (Figure 4(b)). Furthermore, the expression of rFVII in Leishmania cells was determined by SDS-PAGE, and western blot analyses. Expressed protein was run onto $12 \%$ (V/V) SDS-PAGE and the protein band about $55 \mathrm{KD}$ was detected which indicates protein expression of rFVII as shown in Figure 5(a). 


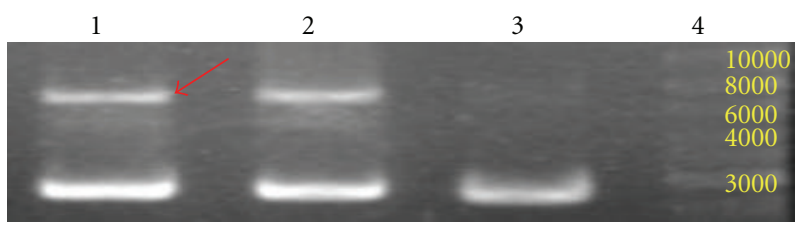

(a)

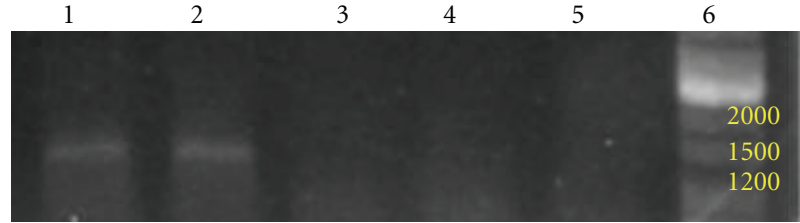

(b)

FIGURE 4: (a) Linearized pLEXSY-hyg2-FVII construct with SwaI (Lanes 1, 2), control plasmid (Lane 3). The arrow head shows a 6500-bp fragment containing cDNA sequence encoding human FVII, hygromycin B resistance gene, $5^{\prime}$ and $3^{\prime}$ portion of the small subunit of $L$. tarentolaer RNA gene ( $5^{\prime}$ and $3^{\prime}$ ssu) and three UTRs: utr1, utr2, and utr3. (b) Confirmation of FVII expression by transfected Leishmania cells Lanes (1,2), Control cells (Lanes 3, 4), Control plasmid (Lane 5).

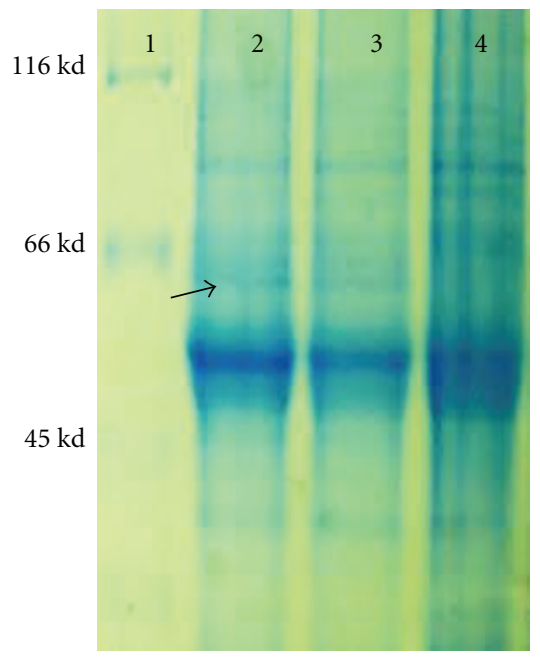

(a)

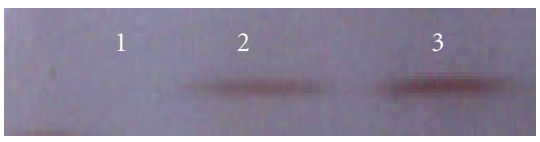

(b)

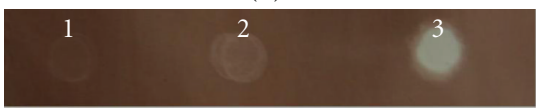

(c)

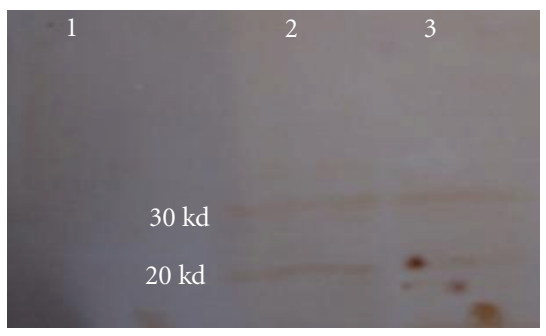

(d)

FIGURE 5: Analysis of expression of the human FVII by SDS-PAGE and western blotting and detection of purified rFVII by dot blot and western blot. (a) FVII protein band on 12\% SDS-PAGE from transfected (Lanes 2, 3) and untransfected I.L.L. as a negative control (Lane 4). (b). expressed FVII protein band in transfected I.L.L. (Lane 2) and negative control untransfected cells (Lane 1), commercially available rFVII as a positive control (Lane 3). (c) Positive control sample rFVIIa (Lane 1), purified rFVII appears as a brown colored spot (Lane 2), purified product of negative control untransfected cells. (d) the purified rFVII from transfected I.L.L (Lane 2, 3) was analyzed by western blot. Two chains of activated form of rFVII with corresponding length are shown; Lane 1, untransfected I.L.L.

The protein identity was verified by western blot analysis. The assay revealed specifically recognition of rFVII coding gene by human FVII antibody (a sheep polyclonal antibody to human FVII, IgG HRP-conjugated). The results were compared with the positive control sample FVII (FVIIa, US Biological), and no reactivity was observed with negative control cells (Figure 5(b)).

Purification of rFVII was achieved using affinity column and by passing a cell lysate of Leishmania over aCNBractivated sepharose 4B resin coupled to specific factor VII polyclonal antibody. The presence of purified rFVII was confirmed by visualizing a brown spot in a dot blot membrane against specific polyclonal antibody (Figure 5(c)).

Furthermore, western blot analysis of the purified rFVII revealed that, during purification, it was converted into the two-chain-activated form (Figure 5(d)).

The resulting two chains were light and heavy chains with a molecular weight of $\sim 20 \mathrm{KDa}$ and $\sim 30 \mathrm{KDa}$, respectively. The purified rFVII concentration was estimated to be about $10 \mu \mathrm{g}$ per $10 \mathrm{~mL}$ of culture medium.
Dialyzed rFVII was added to human plasma immunodepleted of factor VII, and coagulation times were determined. The biological activity of dialyzed rFVII from I.L.L. in comparison with FVII (FVIIa, US Biological) was performed. In the initial experiments, a reduction in clotting time of about $(33 \pm 3)$ seconds with the purified rFVII was observed (Figure 6).

Also, comparison between different dilutions of dialyzed rFVII in a similar assay with standard curve indicated that dialyzed rFVII (rFVIIa) at dilution of (1/1) retained approximately $9 \%$ of the activity of Unicalibrator with $100 \%$ FVIIa level (Figure 7).

\section{Discussion}

In recent years, there has been increasing interest in the use of recombinant activated factor VII (rFVIIa) as an adjunct to the coagulation process. rFVIIa is a useful treatment option for patients with congenital or acquired hemophilia. The findings represent significant advancement 


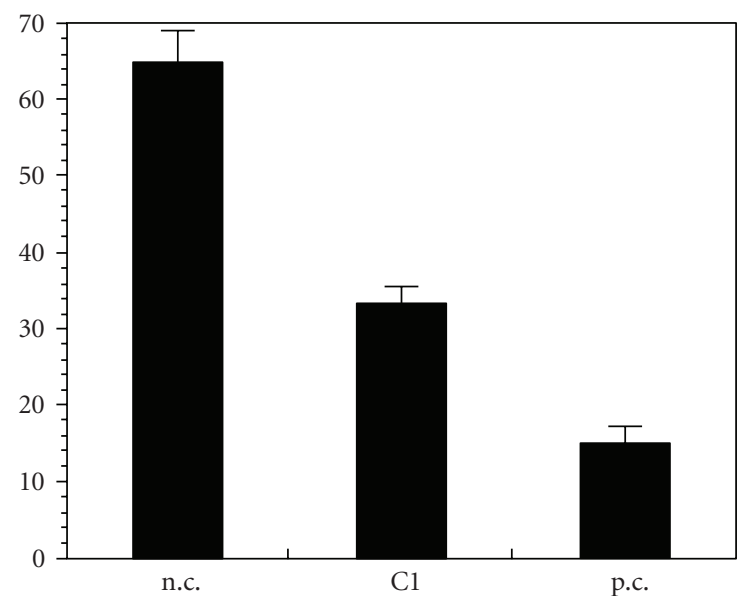

Figure 6: Prothrombin time assay. PT assay was performed for determination of coagulant activity of dialyzed rFVII. Coagulation time measured for dialyzed rFVII produced from transfected I.L.L. (C1: clone_1), commercially available rFVIIa as a positive control (p.c.), purified sample obtained from untransfected I.L.L. as a negative control (n.c.). A reduction in clotting time of about (33 \pm 3 ) seconds with the purified rFVII was observed. Data are shown as mean $\pm \mathrm{SD}$.

in the management of patients who produce antibodies against FVIII or FIX [7].

Initially, BHK cells and subsequently the variety of recombinant protein expression systems such as insect and other mammalian cells have been developed as a resource of FVII gene expression $[4,10,11]$. However, in this study, leishmania expression system was used as a host cell.

I.L.L. cells were transfected with the vector pLexsy-hFVII, which encoded a full-length recombinant human FVII of 406 amino acids, and was integrated properly between $5^{\prime}$ and $3^{\prime}$ portion of the small subunit of rRNA gene and powerfully transcribed by RNA polymerase I of Leishmania cells. Interestingly, gene regulation, in Trypanosomatidae, occurs predominantly after transcription through intergenic untranslated regions (UTRs) [25]. Therefore, suitable UTRs should be chosen. The origins of UTRs of pLEXSY-hyg2 expression vector are as follows: UTR1, $5^{\prime}$ nontranslated region of aprt gene of Leishmania with splice acceptor site for target gene; UTR2, $1.4 \mathrm{~kb}$ intergenic region from cam operon of Leishmania with polyA site for target gene and splice acceptor site for marker gene; UTR3, 5' UTR of dhfr-ts gene of Leishmania with polyA site for marker gene. Results of this study are also in agreement with earlier observations that Leishmania species are able to produce heterologous proteins.

By using native human signal sequence, Breitling et al. [14] could obtain the clone with the maximum expressionproduced recombinant erythropoietin at approximately $30 \mathrm{mg} / \mathrm{L}$ of suspension culture.

In this study, the final yield of the purified rFVII was estimated to be about $1 \mu \mathrm{g} / \mathrm{mL}$ of culture medium. This yield was comparable to that of rFVII-producing mammalian and insect cell lines [10, 26, 27]. Furthermore, it has been demonstrated that Leishmania tarentolae is able to produce

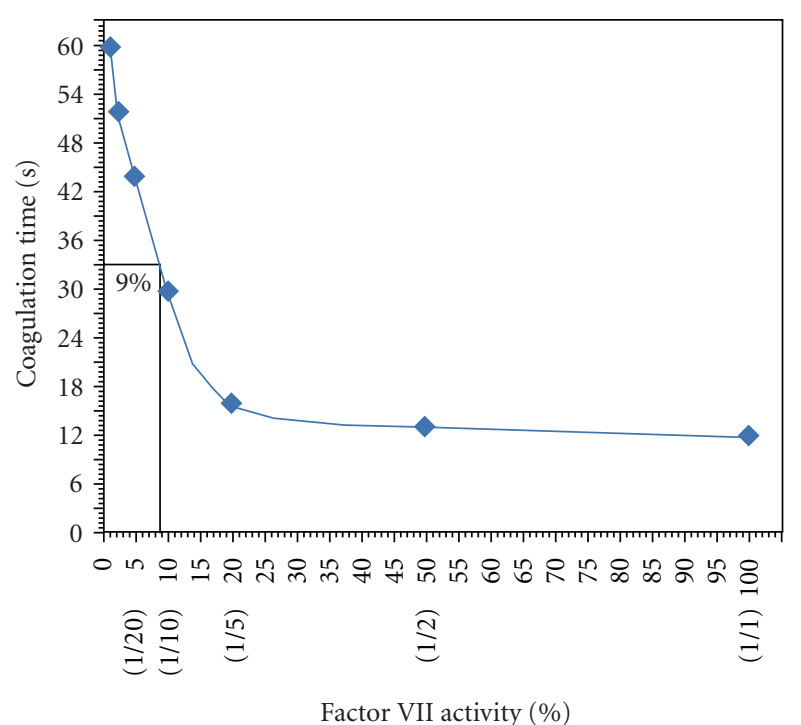

FIGURE 7: Estimation of the yield of functional rFVII (rFVIIa) by one-stage clotting assay (PT-based assay for FVII). Standard curve of different dilutions of unicalibrator was made. Also, clotting times of dilutions of $(1 / 1),(1 / 2),(1 / 5),(1 / 10)$ of dialyzed rFVII were recorded. The clotting times of dilutions of dialyzed rFVII were $33,42,44$, and 48 (s), respectively. The black line mark the (1/1) dilution of dialyzed rFVII activity at the point of intersection of the standard curve through this the yield of functional rFVII (rFVIIa) was estimated $9 \%$ of Unicalibrator with $100 \%$ FVIIa level. The clotting time of the negative control sample (without FVII) was 60 (s).

mammalian-like biantennary N-glycans [28]. So, according to the estimated molecular weight of rFVII produced by Leishmania cells and its comparison with carefully selected positive control of FVII (FVIIa, US Biological), it is thought that $\mathrm{N}$-glycosylation profile and probably the protein folding of this product are similar to those in mammalian cells [29].

Before purification of rFVIIa, electrophoretic and western blot analysis indicated that it consisted of a single band with an estimated molecular weight of $55 \mathrm{kDa}$ on SDS-PAGE. It indicates that rFVII has been synthesized as a singlechain form and then cleaved to the biologically active form during protein purification. Western blot analysis indicated that the resultant active form is composed of light and heavy chains with a molecular weight of $\sim 20 \mathrm{KDa}$ and $\sim 30 \mathrm{KDa}$, respectively [30].

The results of PT assay showed a reduction in clotting time of about 30 seconds with the purified rFVII. As demonstrated by comparing clotting time of the dialyzed rFVII with standard curve, the yield of functional rFVII (rFVIIa) was estimated $9 \%$. The specific findings with this product may be related to its posttranslational modification like $\gamma$ carboxylation. And it should be noted that considerably more investigations are required in order to determine posttranslational $\gamma$-carboxylation of glutamic acid residues in Gla domain of this product in Leishmania cells.

In conclusion, this study has demonstrated for the first time that Leishmania cells can be used as a powerful host for production of recombinant human FVII. The main 
advantages of this expression system are its easy handling like E. coli and yeast expression systems, proper protein folding, posttranslation modification and cultivation in lowcost media such as LB.

\section{Acknowledgments}

This study was carried out at the Cellular and Molecular Biology Research Center of Shahid Beheshti University of Medical Sciences, Tehran, Iran. The authors are thankful for the support and encouragement provided by the staff and management of this Center.

\section{References}

[1] B. Osterud and S. I. Rapaport, "Activation of factor IX by the reaction product of tissue factor and factor VII: additional pathway for initiating blood coagulation," Proceedings of the National Academy of Sciences of the United States of America, vol. 74, no. 12, pp. 5260-5264, 1977.

[2] F. S. Hagen, C. L. Gray, and P. O'Hara, "Characterization of a cDNA coding for human factor VII," Proceedings of the National Academy of Sciences of the United States of America, vol. 83, no. 8, pp. 2412-2416, 1986.

[3] A. C. W. Pike, A. M. Brzozowski, S. M. Roberts, O. H. Olsen, and E. Persson, "Structure of human factor VIIa and its implications for the triggering of blood coagulation," Proceedings of the National Academy of Sciences of the United States of America, vol. 96, no. 16, pp. 8925-8930, 1999.

[4] F. Hagen, M. Murray et al., "Expression of factor VII activity in mammalian cells," U.S. Patent, no. 4, pp. 784-950, 1988.

[5] U. Hedner and C. A. Lee, "First 20 years with recombinant FVIIa (NovoSeven)," Haemophilia, vol. 17, no. 1, pp. e172e182, 2011.

[6] R. B. Weiskopf, "Recombinant-activated coagulation factor VIIa (NovoSeven): current development," Vox Sanguinis, vol. 92, no. 4, pp. 281-288, 2007.

[7] A. Mayo, M. Misgav, Y. Kluger et al., "Recombinant activated factor VII (NovoSeven): addition to replacement therapy in acute, uncontrolled and life-threatening bleeding," Vox Sanguinis, vol. 87, no. 1, pp. 34-40, 2004.

[8] M. Levi, M. Peters, and H. R. Büller, "Efficacy and safety of recombinant factor VIIa for treatment of severe bleeding: a systematic review," Critical Care Medicine, vol. 33, no. 4, pp. 883-890, 2005.

[9] M. Levi, J. H. Levy, H. F. Andersen, and D. Truloff, "Safety of recombinant activated factor VII in randomized clinical trials," New England Journal of Medicine, vol. 363, no. 19, pp. 1791-1800, 2010.

[10] N. Masroori, R. Halabian, M. Mohammadipour et al., "Highlevel expression of functional recombinant human coagulation factor VII in insect cells," Biotechnology Letters, vol. 32, no. 6, pp. 803-809, 2010.

[11] R. Halabian, M. H. Roudkenar, N. S. Esmaeili, N. Masroori, A. M. Roushandeh, and A. J. Najafabadi, "Establishment of a cell line expressing recombinant factor VII and its subsequent conversion to active form FVIIa through hepsin by genetic engineering method," Vox Sanguinis, vol. 96, no. 4, pp. 309315, 2009.

[12] R. Sodoyer, "Expression systems for the production of recombinant pharmaceuticals," BioDrugs, vol. 18, no. 1, pp. 51-62, 2004.
[13] F. Hesse and R. Wagner, "Developments and improvements in the manufacturing of human therapeutics with mammalian cell cultures," Trends in Biotechnology, vol. 18, no. 4, pp. 173$180,2000$.

[14] R. Breitling, S. Klingner, N. Callewaert et al., "Non-pathogenic trypanosomatid protozoa as a platform for protein research and production," Protein Expression and Purification, vol. 25, no. 2, pp. 209-218, 2002.

[15] C. Fritsche, M. Sitz, N. Weiland, R. Breitling, and H. D. Pohl, "Characterization of the growth behavior of Leishmania tarentolae-a new expression system for recombinant proteins," Journal of Basic Microbiology, vol. 47, no. 5, pp. 384-393, 2007.

[16] C. Fritsche, M. Sitz, M. Wolf, and H. D. Pohl, "Development of a defined medium for heterologous expression in Leishmania tarentolae," Journal of Basic Microbiology, vol. 48, no. 6, pp. 488-495, 2008.

[17] B. Kazemi, G. Tahvildar, S. R. H. Feshareki, and E. E. Javadian, "Isolation a lizard Leishmania promastigote from natural host in Iran," Journal of Biological Sciences, vol. 4, no. 5, pp. 620623, 2004.

[18] S. Koizume, M. S. Jin, E. Miyagi et al., "Activation of cancer cell migration and invasion by ectopic synthesis of coagulation factor VII," Cancer Research, vol. 66, no. 19, pp. 9453-9460, 2006.

[19] W. Gaastra and K. Hansen, "Ligation of DNA with $t_{4}$ DNA ligase," Methods in Molecular Biology, vol. 2, pp. 225-230, 1985.

[20] D. Hanahan, "Studies on transformation of Escherichia coli with plasmids," Journal of Molecular Biology, vol. 166, no. 4, pp. 557-580, 1983.

[21] K. A. Robinson and S. M. Beverley, "Improvements in transfection efficiency and tests of RNA interference (RNAi) approaches in the protozoan parasite Leishmania," Molecular and Biochemical Parasitology, vol. 128, no. 2, pp. 217-228, 2003.

[22] M. Soleimani, F. Mahboudi, N. Davoudi et al., "Expression of human tissue plasminogen activator in the trypanosomatid protozoan Leishmania tarentolae," Biotechnology and Applied Biochemistry, vol. 48, no. 1, pp. 55-61, 2007.

[23] H. P. Phan, M. Sugino, and T. Niimi, "The production of recombinant human laminin-332 in a Leishmania tarentolae expression system," Protein Expression and Purification, vol. 68, no. 1, pp. 79-84, 2009.

[24] R. Toso, M. Pinotti, K. A. High, E. S. Pollak, and F. Bernardi, "A frequent human coagulation Factor VII mutation (A294V, c152) in loop 140s affects the interaction with activators, tissue factor and substrates," Biochemical Journal, vol. 363, no. 2, pp. 411-416, 2002.

[25] E. Handman, "Cell biology of Leishmania," Advances in Parasitology, vol. 44, pp. 1-39, 1999.

[26] S. M. Ruiz, S. Sridhara, M. A. Blajchman, and B. J. Clarke, "Expression and purification of recombinant rabbit factor VII," Thrombosis Research, vol. 98, no. 2, pp. 203-211, 2000.

[27] N. Wajih, J. Owen, and R. Wallin, "Enhanced functional recombinant factor VII production by HEK 293 cells stably transfected with VKORC1 where the gamma-carboxylase inhibitor calumenin is stably suppressed by shRNA transfection," Thrombosis Research, vol. 122, no. 3, pp. 405-410, 2008.

[28] A. J. Parodi, "N-Glycosylation in trypanosomatid protozoa," Glycobiology, vol. 3, no. 3, pp. 193-199, 1993.

[29] G. Bolt, C. Kristensen, and T. D. Steenstrup, "Posttranslational $\mathrm{N}$-glycosylation takes place during the normal processing of human coagulation factor VII," Glycobiology, vol. 15, no. 5, pp. 541-547, 2005. 
[30] P. Margaritis, V. R. Arruda, M. Aljamali, R. M. Camire, A. Schlachterman, and K. A. High, "Novel therapeutic approach for hemophilia using gene delivery of an engineered secreted activated Factor VII," Journal of Clinical Investigation, vol. 113, no. 7, pp. 1025-1031, 2004. 

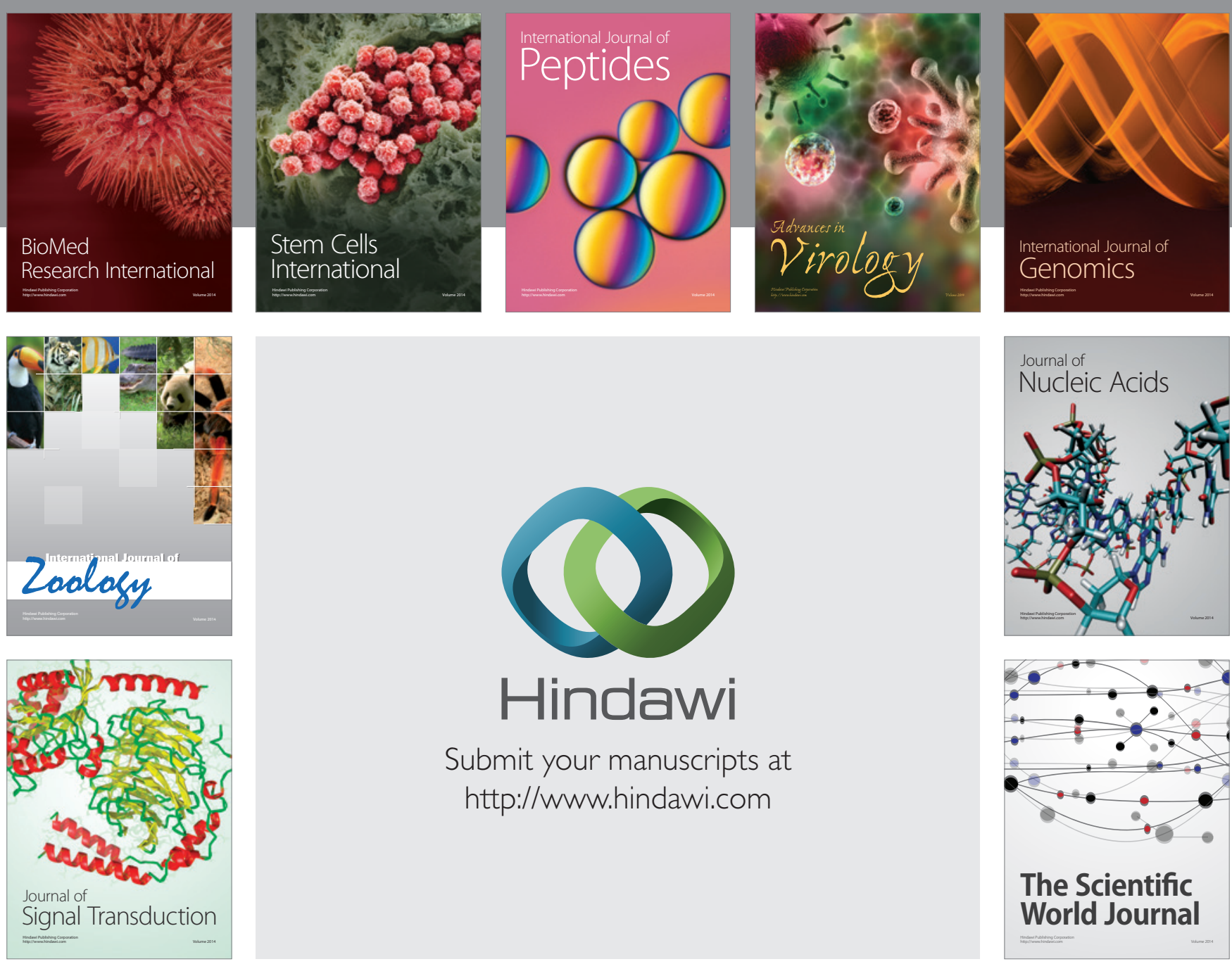

Submit your manuscripts at

http://www.hindawi.com
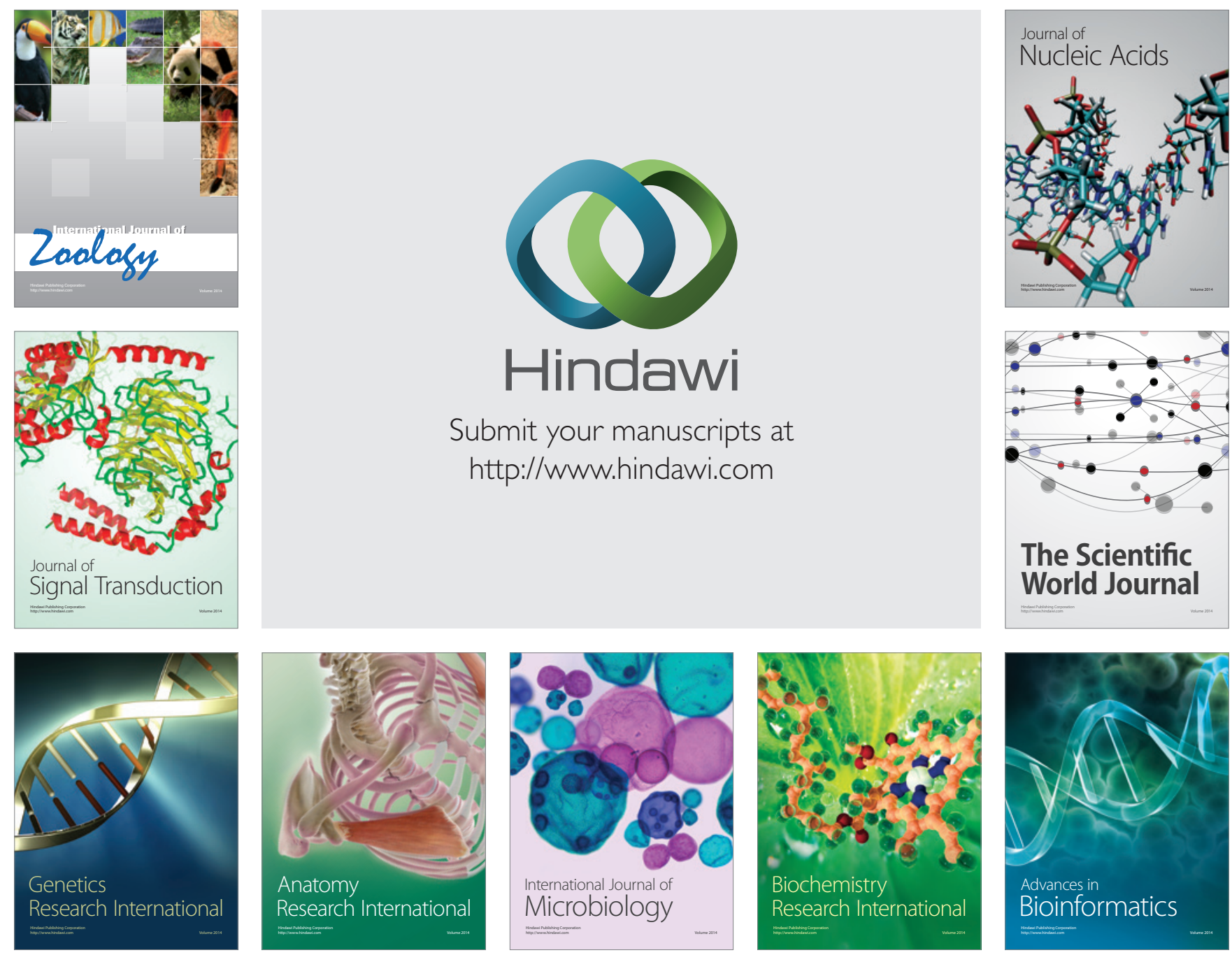

The Scientific World Journal
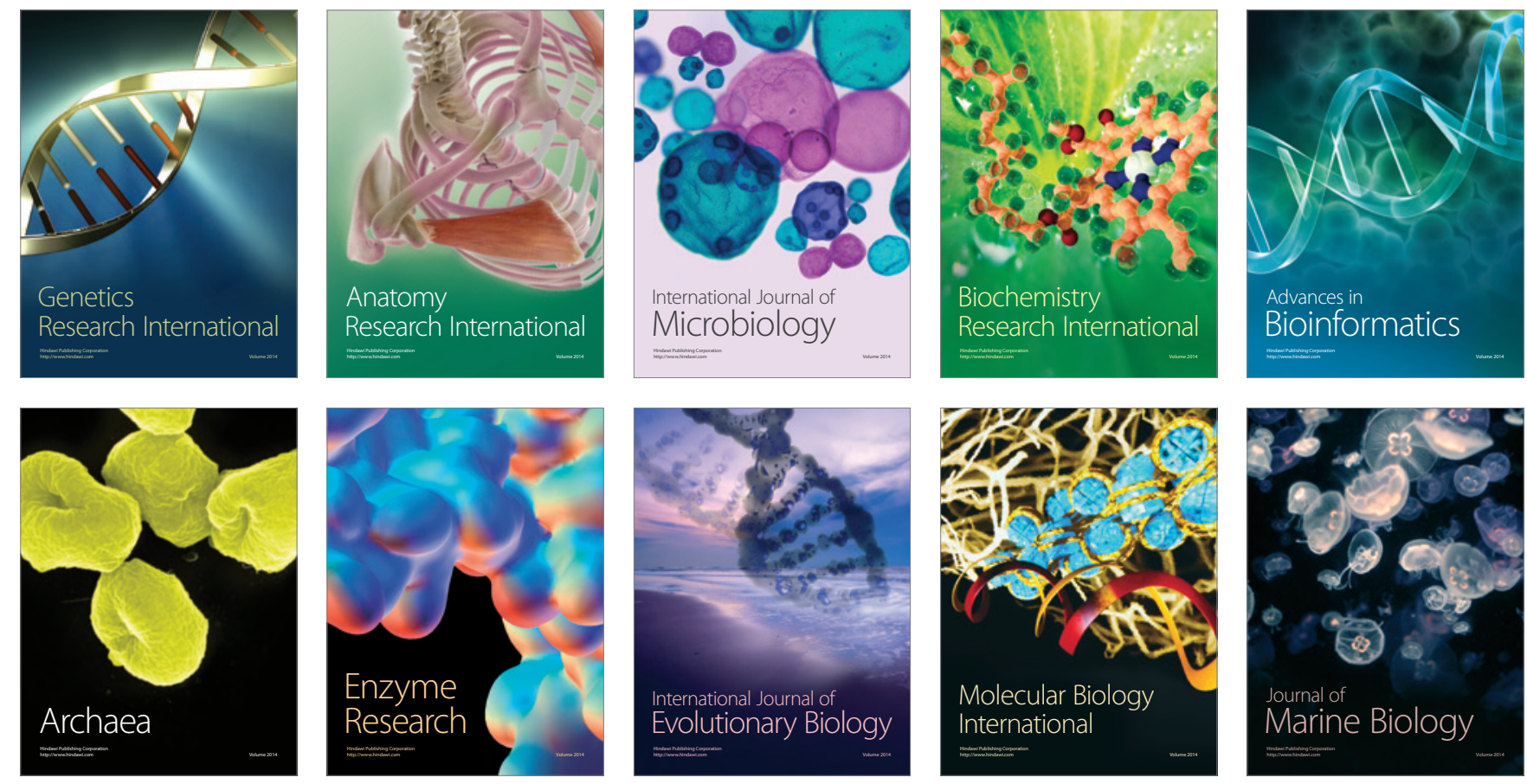